\title{
DYNAMICAL EXPERIMENTS ON MODELS OF COLLIDING DISK GALAXIES
}

\author{
Richard A. Gerber,* Dinshaw S. Balsara, ${ }^{\dagger}$ and Susan A. Lamb* ${ }^{\dagger}$
}

\section{Introduction.}

Collisions between galaxies can induce large morphological changes in the participants and, in the case of colliding disk galaxies, bridges and tails are often formed. Observations of such systems indicate a wide variation in color (see Larson and Tinsley, 1978) and that some of the participants are experiencing enhanced rates of star formation, especially in their central regions (Bushouse 1986, 1987; Kennicutt et al., 1987, Bushouse, Lamb, and Werner, 1988). In this paper we describe progress we have made in understanding some of the dynamics of interacting galaxies using N-body stellar dynamical computer experiments, with the goal of extending these models to include a hydrodynamical treatment of the gas so that a better understanding of globally enhanced star formation will eventually be forthcoming.

Historically, N-body experiments have been used by a number of authors to study galactic collisions (eg. Toomre and Toomre, 1972; Miller and Smith, 1980; Noguchi, 1987). Our results, which were obtained using a three-dimensional grid-based Fast Fourier Transform N-body program, confirm the earlier results and lay the foundation for an explicit investigation of the gas dynamics in such systems. We are especially interested in eventually building models that will address the phenomenon of enhanced star formation rates and we plan to incorporate a representation of galactic gas into the computer models. We plan to follow the galactic gas continuum, looking for regions of enhanced gas density and locations of shocks. We will be using Balsara's Smoothed Particle Hydrodynamics code (Balsara and Norman, 1990), which includes the $\mathrm{N}$-body code, to model both the stellar and gaseous components of disk galaxies during a collision.

We concentrate our present N-body study on the first stages of the interaction, that is the first flyby. We anticipate the both stars and any gas present in the galaxies will respond similarly to changes in the gravitational potential in the early stages of a collision. Only when the dissipative nature of the gas becomes important, as when shock fronts appear, will gas and star motions possibly decouple to a great extent. Thus we can obtain a first approximation to the locations of possible star formation by studying the first stages of the collision. 


\section{Model Parameters and Results}

We model collisions between a spiral galaxy and an elliptical galaxy. The spiral galaxy is represented by a rotationally supported exponential disk of stars, stabilized by both internal velocity dispersions (Toomre $\mathrm{Q}=1.5$ ) and a surrounding massive halo. The halo, with a mass three times that of the disk, is a spherical King Model (King, 1966) that was evolved as the disk potential was slowly grown within it, producing our starting disk galaxy model. The second galaxy, which represents a spherical galaxy, is a King Model with a mass equal to the halo mass in the spiral galaxy. There were approximately 55,000 particles used in each of the experiments, which were run on the Cray-2 supercomputer at the National Center for Supercomputing Applications at the University of Ilinois at Urbana-Champaign.

We considered four disk/orbital orientations, all with an initial value of orbital energy at which two point masses would be on parabolic orbits (ie. zero total orbital energy). The four experiments are:

1) Prograde (orbital and disk rotational angular momentum vectors parallel), grazing (minimum center of mass separation of approximately two disk radii).

2) Retrograde (orbital and disk rotational angular momentum vectors antiparallel), grazing

3) Head on (zero orbital angular momentum), disk lying in orbital plane.

4) Head on, disk normal to orbital plane.

We choose to scale our experiments such that the spiral galaxy has parameters similar to those of our Galaxy. In these units, the disk has an exponential scale length of $4 \mathrm{kpc}$ and both the disk and halo have similar outer radii of approximately $20 \mathrm{kpc}$. The disk's mass is $6 \times 1010 \mathrm{MO}$ and the halo has a mass of $1.8 \times 10^{11} \mathrm{Mo}$. The disk has a thickness of about $500 \mathrm{pc}$ and its rotational velocity at $8 \mathrm{kpc}$ is $230 \mathrm{~km} / \mathrm{sec}$.

\section{Prograde, in disk plane}

The disk is violently affected in this first experiment. It has been known since the experiments of Toomre and Toomre (1972) that prograde collisions produce a great deal of damage, and indeed particles are sprayed in both the forward and backward directions; some stars appear to be captured by the elliptical galaxy, hinting at the possibility that gas as well as stars may be exchanged via this process. We note the formation of a bar-like structure in the central region of the disk galaxy. We also measure a global outward average radial streaming of disk particles at a maximum of 60 $\mathrm{km} / \mathrm{sec}$. As these velocities are greater than the sound speed and the structure is very nonaxisymmetric (indicating a nonhomologous response), we expect that shocking will occur. An 
example of the resulting morphology is given in figure 1, which contains a sampling of the positions of the 'stellar' particles at time 120 million years after closest approach.

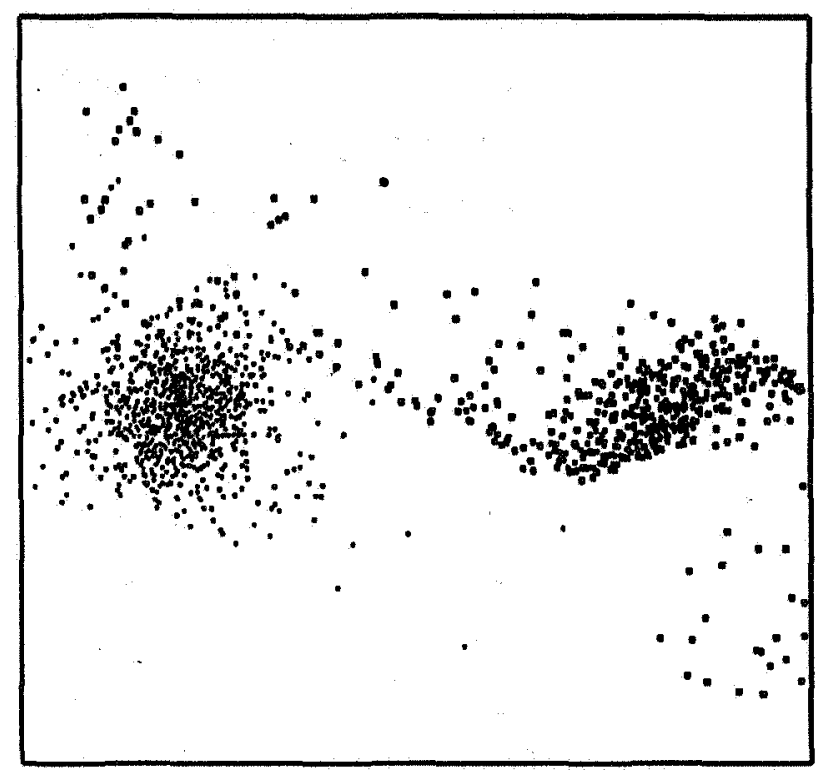

Figure 1

The morphology of the disk and elliptical galaxies for experiment 1 (prograde, grazing collision) is shown. The larger dots represent disk particles and the smaller dots represent the elliptical galaxy. The disk galaxy's halo particles are not shown. The time is $\mathbf{1 2 0}$ million years after closest approach. The disk system is moving to the right and upward while the elliptical is moving left and downward. The view is looking down onto the disk plane.

\section{Retrograde, in disk plane}

The retrograde collision exhibits drastically different behavior than that shown by the prograde experiment. The disk is only slightly affected in the retrograde encounter, generally maintaining its axisymmetric shape while expanding slightly. We find only very small average radial streaming velocities in this case. That such a close encounter produces such small effects may be at least part of the explanation of Bushouse's (1986) finding that a substantial proportion ( $\approx 30$ percent) of closely interacting pairs of disk galaxies show no evidence for enhanced star formation rates.

\section{Head on, in disk plane.}

A striking effect of this deeply interpenetrating collision is the sharp contraction of both disk and halo components soon after closest approach. In contrast to the grazing collisions, large inward velocity flows are triggered, reaching an average maximum value of $100 \mathrm{~km} / \mathrm{sec}$ in the disk. All 
galactic components contract to nearly one-half their previous size and then later re-expand as material flows outward at a peak average velocity of $90 \mathrm{~km} / \mathrm{sec}$. The disk is quite disrupted with a definite, although transient, thin bar-like feature forming in the center. In real interactions of this type there may be a sizeable buildup of gas in the center of the disk galaxy due to dissipative interactions (such as shocking and high rates of cooling in dense regions) in the gas that flows into the center. That is, dissipation may halt the subsequent outward bounce of the gas that would otherwise follow the contraction of the gas and stars.

\section{Head on, normal to disk plane}

This collision produces a striking ring of disk particle soon after close passage. The disk and halo both contract and expand as in the previous experiment; with radial velocities in the disk having maximum inflow and outflow values on the order of $100 \mathrm{~km} / \mathrm{sec}$. These are illustrated in figure 2. A ring forms during the re-expansion phase and this strong density enhancement propagates outward through the disk attaining a peak amplitude approximately 4 times greater than the unperturbed disk stellar surface density. A time sequence showing the evolution of the density profile of the disk is shown in figure 3 and we see there that the enhancement damps significantly by the time it reaches the edge of the disk.

\section{Disk Velocity Components: Face On Collision}

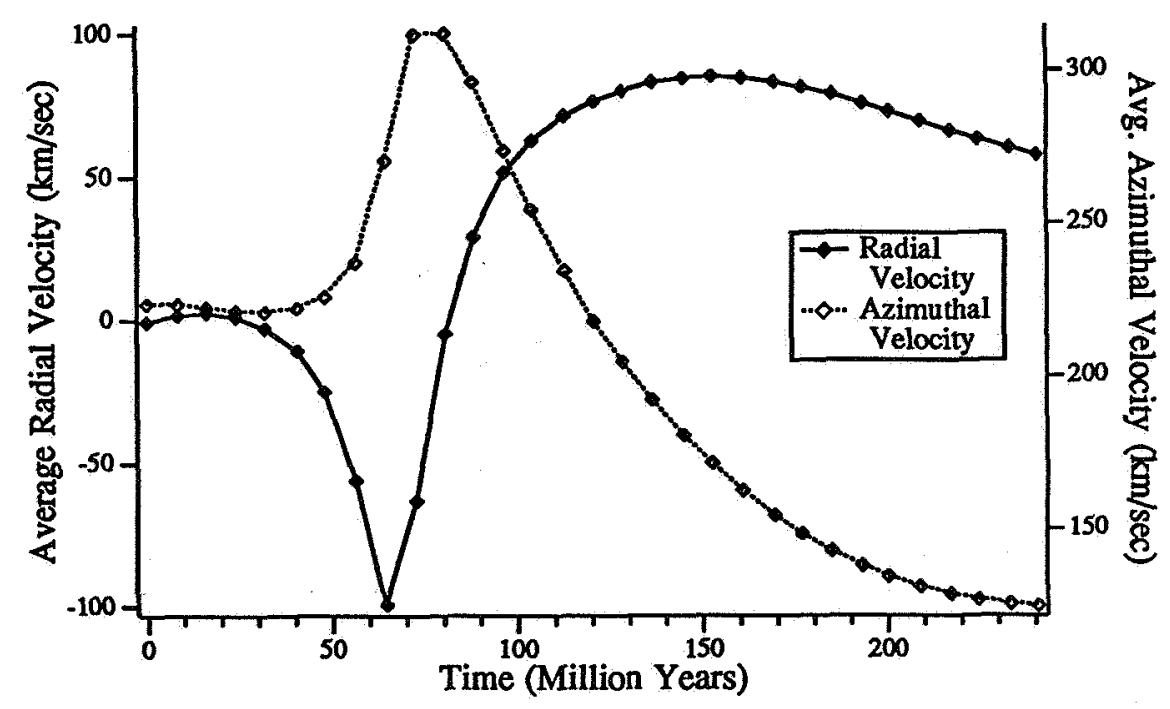

Figure 2

Globally averaged radial and azimuthal velocities as a function of time for the disk particles in experiment 4. 


\section{Particles per Radial Bin: Normal to Disk Plane Collision}
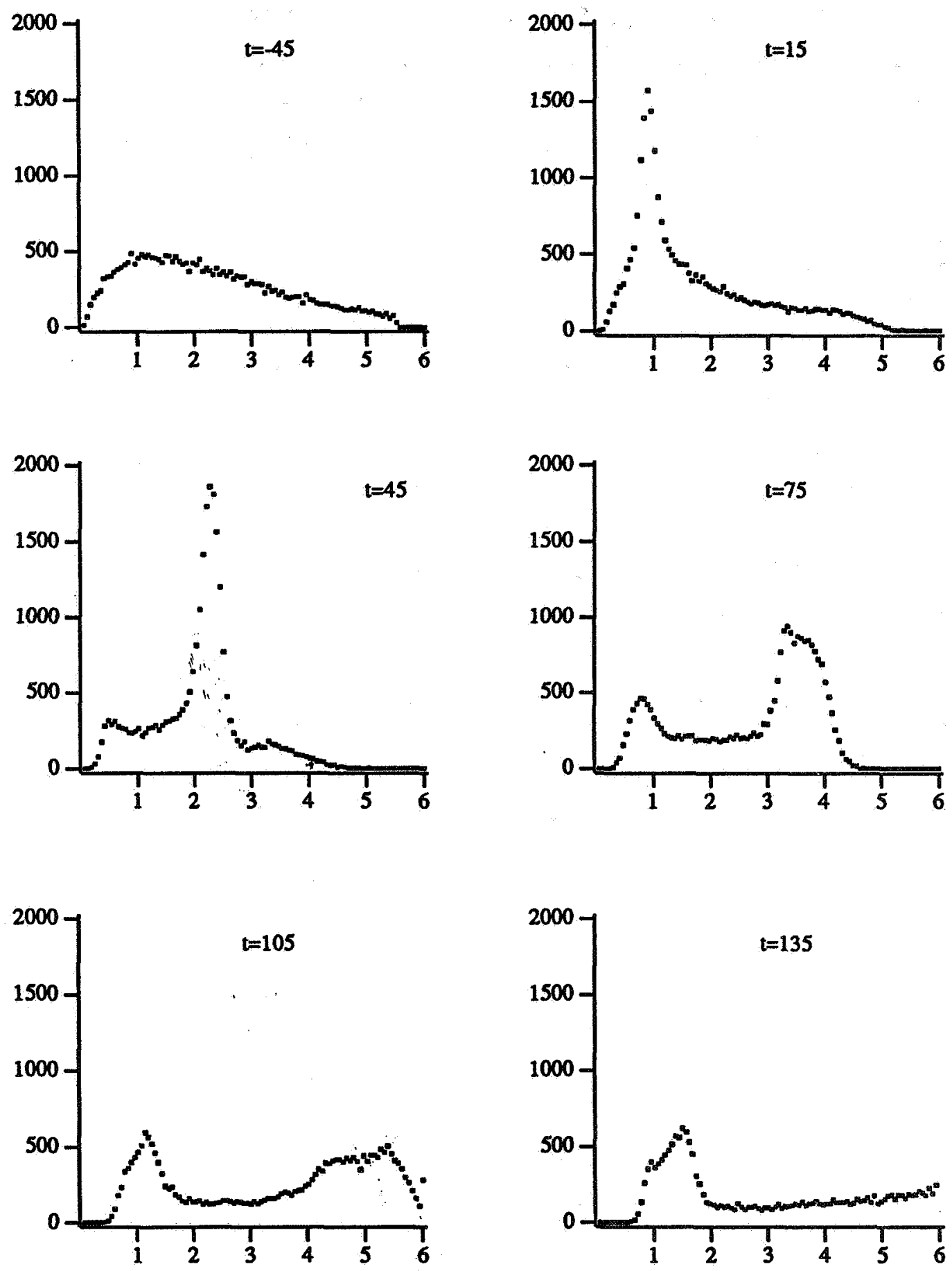

Figure 3

The number of particles per spherical bin at six different times in experiment 4 (the collision normal to the disk plane). The bins are spaced at constant radial intervals; each is centered on the system's center of mass. Time, $t$ (given in million years) is measured relative to closest approach. 


\section{Conclusion}

Close interactions between galaxies can produce large perturbations in both density and velocity fields. We have measured, via computational experiments that represent a galaxy's stars, average radial velocity flows as large as $100 \mathrm{~km} / \mathrm{sec}$ and 400 percent density increases. These can occur in rings that move outwards through the disk of a galaxy, in roughly homologous inflows toward the nucleus, and in off center, non-axisymmetric regions. Here we have illustrated where the gas is likely to flow during the early stages of interaction and in future work we plan to investigate the fate of the gas more realistically by using an N-body/Smoothed Particle Hydrodynamics code to model both the stellar and gaseous components of a disk galaxy during a collision. Specifically, we will determine the locations of enhanced gas density and the strength and location of shock fronts that form during the interaction.

\section{References}

Balsara, D.S. and Norman, M.L. 1990, Submitted to Ap. J.

Bushouse, H.A. 1986, Astron. J., 91, 255.

Bushouse, H.A. 1987, Ap. J., 320, 49.

Bushouse, H.A., Lamb, S.A., and Werner, M.W., 1988, Ap. J., 325, 74.

Kennicutt, R.C., Keel, W.C., van der Hulst, J.M., Hummell, E. and Roettiger, K. 1987, Astron. J., 93, 1011.

King, I.E. 1966, Astron. J., 71, 64.

Larson, R.B. and Tinsley, B.M. 1978, Ap. J., 219, 46.

Miller, R.H. and Smith, B.F. 1980, Ap. J., 223, 122.

Noguchi, M. 1987, Mon. Not. R. astr. Soc., 228, 635.

* Department of Physics, University of Illinois , Urbana, Illinois 61801.

† Department of Astronomy, University of Illinois , Urbana, Illinois 61801. 